\title{
Hypertension, antihypertensive medication use, and breast cancer risk in the California Teachers Study cohort
}

\author{
Joan A. Largent • Leslie Bernstein • Pamela L. Horn-Ross • Sarah F. Marshall • \\ Susan Neuhausen • Peggy Reynolds • Giske Ursin • Jason A. Zell • \\ Argyrios Ziogas • Hoda Anton-Culver
}

Received: 14 October 2009/Accepted: 18 May 2010/Published online: 6 June 2010

(C) The Author(s) 2010. This article is published with open access at Springerlink.com

\begin{abstract}
Background We investigated the association between hypertension, antihypertensive $(\mathrm{AH})$ medication use, and breast cancer in a large prospective study, the California Teachers Study (CTS).

Methods Information on history of hypertension and lifetime regular use of $\mathrm{AH}$ medications was collected from 114,549 women in 1995-1996. Among them, 4,151 invasive breast cancers were diagnosed between 1995 and 2006. Additional information on $\mathrm{AH}$ use was collected from 73,742 women in 2000-2001, and 1,714 of these women were subsequently diagnosed with breast cancer. Cox proportional hazards regression was used to estimate
\end{abstract}

J. A. Largent $(\square)$ - S. F. Marshall · J. A. Zell · A. Ziogas ·

H. Anton-Culver

Department of Epidemiology, College of Health Sciences,

University of California Irvine, 224 Irvine Hall, Irvine,

CA 92697-7555, USA

e-mail: jlargent@uci.edu

L. Bernstein · S. Neuhausen

Department of Population Sciences, City of Hope National

Medical Center, Duarte, CA, USA

P. L. Horn-Ross · P. Reynolds

Northern California Cancer Center, Fremont, CA, USA

P. L. Horn-Ross · P. Reynolds

Division of Epidemiology, Department of Health Research and Policy, Stanford University School of Medicine, Stanford, CA, USA

L. Bernstein · G. Ursin

Department of Preventive Medicine, University of Southern

California Keck School of Medicine, Los Angeles, CA, USA

G. Ursin

Department of Nutrition, University of Oslo, Oslo, Norway relative risks (RR) and 95\% confidence intervals (CI) for breast cancer.

Results Use of AH medication for $\geq 5$ years, when compared with no use, was associated with a modest increased risk of invasive breast cancer $(\mathrm{RR}=1.18$, 95\% CI 1.02-1.36). This increased risk appeared to be confined to estrogen receptor (ER)-positive tumors $(\mathrm{RR}=1.21,95 \% \mathrm{CI} 1.03-1.43)$ and pre-/peri-menopausal women $(\mathrm{RR}=1.58,95 \% \mathrm{CI} 1.11-2.25)$.

Conclusions Increased risk of invasive breast cancer was observed for long-term ( $\geq 5$ years) AH use, and this appeared to be confined to ER + breast cancer and younger women.

Keywords Hypertension - Breast cancer .

Antihypertensive medication

\section{Introduction}

Breast cancer remains an important public health concern as it represents the most common cancer diagnosis and second leading cause of cancer-related death among women in the United States [1,2]. Obesity and obesityrelated conditions including hypertension, a highly prevalent condition among adult women, may be contributing to the burden of post-menopausal breast cancer. A report from the National Health and Nutrition Examination Survey (NHANES III) found that $22 \%$ of all U.S. females 18 years and older were hypertensive or taking $\mathrm{AH}$ medications in 1988-1991 [3].

Hypertension and AH medications have been consistently associated with other malignancies, including renal [4] and endometrial cancers [5], and with benign conditions including uterine fibroids [6]. However, results of studies 
examining the association between breast cancer, hypertension, and AH medications have been inconsistent [7-9]. It has been suggested that the previously observed associations may be explained by uncontrolled confounding from shared risk factors, such as increased obesity, a fat-rich diet, or physical inactivity, alcohol use, and the absence of lactation [10]. However, as both conditions may share common pathophysiologic pathways involved in hormone synthesis and metabolism [11, 12] and growth factor signaling [13], it is possible that a biologic association exists.

Because hypertension is a highly prevalent condition and $\mathrm{AH}$ medications are among the most commonly prescribed drugs in the United States, the importance of examining occurrence of any serious side effects including influences on the risk of breast cancer has been suggested [14]. Therefore, we sought to elucidate the relationship between hypertension, AH medication use, and breast cancer risk in a large prospective cohort of women, the California Teachers Study (CTS).

\section{Methods}

\section{Study population and data collection}

The CTS is a prospective cohort study of 133,479 California female teachers and school administrators who completed a baseline survey in 1995-1996. A detailed description of the study design and methods has been published previously [15]. From among 118,262 participants who were residents of California at baseline and had no prior history of breast cancer, we excluded members who were over 85 years of age at baseline $(n=1,579)$ or who had unknown history of hypertension or AH medication use $(n=2,134)$. The resulting cohort for the analysis of data items collected at baseline consisted of 114,549 women. Women in this subcohort have been followed for breast cancer incidence as well as other health outcomes from the completion of the baseline survey through December 31, 2006, their dates of death or the date of a move out of California for more than four months, whichever came first. Incidence of invasive breast cancer was ascertained through linkage with the statewide population-based California Cancer Registry (CCR). During follow-up, 4,151 women were diagnosed with invasive breast cancer. National Cancer Institute designated Surveillance, Epidemiology, and End Results (SEER) registries, including the CCR, routinely collect estrogen receptor (ER) as recorded in the medical records following a breast cancer diagnosis. ER status was available for 3,572 $(86.0 \%)$ of the cases.

The baseline self-administered questionnaire asked cohort members to indicate whether they had a history of high blood pressure and whether they had taken the following medications regularly (defined as at least once a week): "Reserpine (Raudixin, Ser-Ap-Es, Hydropres, Rauwolfia, Metatensin);" "Water pills for high blood pressure (Diuril, Hydrodiuril, Dyazide, etc.);" or "Other high blood pressure medications," and if taken, to indicate duration of use (did not take regularly, or took for less than 1 year, 2 years, 3-4 years, 5-9 years, $10+$ years), and if took regularly, the frequency of use (1-3 days per week, 4-6 days per week, every day). Duration variables included in the analyses were categorized as follows: Never regular use, less than or equal to one year, 2-4 years, 5-9 years, or $10+$ years. An overall variable "treated hypertension" was created which included women with self-reported history of hypertension and also use of at least one AH medication.

\section{Statistical analysis}

Risk factors included in the analyses as potential confounders were based on the data collected as part of the self-administered baseline questionnaire. These included family history of breast cancer (at least one affected firstdegree relative, no affected first-degree relatives, or unknown/adopted); race/ethnicity (White; African-American; Hispanic; Asian/Pacific Islander; or other/mixed/not specified); body mass index ( $<20 \mathrm{~kg} / \mathrm{m}^{2} ; 20$ to $<25 \mathrm{~kg} / \mathrm{m}^{2}$; 25 to $<29 \mathrm{~kg} / \mathrm{m}^{2} ; 30$ to $<35 \mathrm{~kg} / \mathrm{m}^{2} ; \geq 35 \mathrm{~kg} / \mathrm{m}^{2}$; or unknown); smoking (never smoker; former smoker; current smoker; or unknown); alcohol use in the year prior to baseline, measured in average grams per day (no use; less than 20 grams per day; 20 grams per day or more; or unknown); physical activity, defined as average lifetime (from high school through current age or aged 54 if 55 or older at baseline) moderate or strenuous activity per week, ( $<2 \mathrm{~h} ; 2$ to $<4 \mathrm{~h} ; 4$ to $<6 \mathrm{~h} ; 6$ or more hours; or unknown); a summary parity variable (first full-term pregnancy (FFTP) before age 25; FFTP age 25-29; nulliparous before age 30; or unknown); breastfeeding history (never or less than 12 months; at least 12 months; or unknown); hysterectomy (never; ever; or unknown); menopausal status and hormone therapy use (premenopausal; peri-menopausal; post-menopausal and never used hormone therapy; postmenopausal and ever used hormone therapy; or unknown menopausal status or HT use); diabetes history (ever; or never); and percent calories from fat, excluding alcohol (quartiles). A variable for adherence to screening guidelines was created and defined by age: for women age $\geq 40$ at baseline having a mammogram within 1 year of baseline; for women age $<40$ at baseline having a breast exam by health care provider within 2 years or a mammogram within 1 year of baseline (no; yes; or unknown). This screening adherence variable was tested in the models, and 
as it did not change, RR estimates by at least ten percent was subsequently dropped.

More detailed information on $\mathrm{AH}$ medication use was ascertained in a follow-up self-administered questionnaire mailed to cohort members in 2000-2001. This questionnaire queried daily use of the following $\mathrm{AH}$ medications for at least two months in the two years preceding the survey: "Thiazide diuretic (for example: Diuril, Hydrodiuril, Dyazide);" "Lasix;" "Calcium blocker (for example: Calan, Procardia, Cardizem);" “ACE inhibitors (for example: Capoten, Vasotec, Zestril);" and "Other high blood pressure medication." This questionnaire was completed by 89,058 cohort members. Exclusions for this analysis included women who had moved out of California $(n=8,608)$, who were diagnosed with breast cancer prior to completion of this survey ( $n=5,476$ ), whose history of breast cancer was unknown $(n=54)$, or who did not complete the $\mathrm{AH}$ medication portion of the survey $(n=1,178)$. This analysis was conducted independently of hypertension or AH medication use reported at baseline. These exclusions resulted in 73,742 women eligible for analyses using the 2000-2001 questionnaire data. For these analyses, follow-up was initiated on the date the questionnaire was completed and women were followed until the first of the following: incident breast cancer, death, a move out of California, or December 31, 2006. In this period, 1,714 invasive breast cancers were diagnosed.

Multivariable Cox proportional hazards regression methods were used to estimate the relative hazard (referred to herein as the relative risk, RR) for developing breast cancer during the follow-up period; $95 \%$ confidence intervals (CI) were constructed around the point estimates for the RRs [16] associated with history of hypertension and $\mathrm{AH}$ medication use, using ages at start and end of follow-up (in days) to define time on study. Models included the covariates listed above and were stratified by age at baseline (in single years of age).

All analyses were performed using SAS version 9.2 (SAS Institute Inc., Cary, NC, USA).

\section{Results}

The distributions of selected baseline characteristics and the age-adjusted differences of these factors by hypertension status are described in Table 1. The mean age at baseline was 52.8 , and the majority of women $(86.4 \%)$ were of non-Hispanic White race/ethnicity. A history of high blood pressure was reported by $16.8 \%$ of the women. The most common category of $\mathrm{AH}$ medication use reported on the baseline questionnaire among all women was "other high blood pressure medication" (13.0\% of all women, $68.4 \%$ of hypertensives), followed by diuretics $(10.0 \%$ of all women, $45.3 \%$ of hypertensives $)$ and reserpine $(0.4 \%$ of all women, $2.2 \%$ of hypertensives). Women with a history of hypertension were significantly older and more likely to be non-White, to have a higher body mass index (BMI), to have a history of diabetes, to be nulliparous at age of 30 , to be adherent to breast screening guidelines, to have had a hysterectomy, and to consume a higher percent of calories from fat than women without a history of hypertension. Furthermore, hypertensive women were less likely to have breastfed for 12 months or more or to have engaged in moderate or strenuous physical activity over their lifetimes.

Table 2 presents associations between hypertension, $\mathrm{AH}$ medication use, and breast cancer for both age-adjusted and multivariable-adjusted models for the full cohort, with analyses also presented by ER status. A history of treated hypertension, when compared to either women without hypertension or without $\mathrm{AH}$ medication use was not significantly associated with invasive breast cancer overall or by ER subtype. Long-term use of any $\mathrm{AH}$ medication was associated with increased risk of invasive breast cancer overall $(\mathrm{RR}=1.18,95 \%$ CI 1.02-1.36; $\mathrm{RR}=1.18,95 \%$ CI 1.04-1.34) for 5 and 10+ years of use, respectively). This association appeared to be confined to ER-positive breast cancer $(\mathrm{RR}=1.21,95 \% \mathrm{CI} 1.03-1.43$; $\mathrm{RR}=1.26$, $95 \%$ CI $1.10-1.45$, for 5 and $10+$ years of use, respectively). Ten or more years of diuretic use was also associated with breast cancer overall and ER-positive subtype $(\mathrm{RR}=1.16,95 \%$ CI 1.01-1.33; RR $=1.21,95 \%$ CI 1.03 1.42 , respectively). Only 500 women reported regular use of reserpine, which was not associated with breast cancer risk $(\mathrm{RR}=0.75,95 \% \mathrm{CI} 0.48-1.16)$.

Stratified analyses by categories of BMI $\left(<25 \mathrm{~kg} / \mathrm{m}^{2} ; 25\right.$ to $<30 \mathrm{~kg} / \mathrm{m}^{2}$; and $\geq 30 \mathrm{~kg} / \mathrm{m}^{2}$ ) and menopausal status (pre-/peri-menopausal; and post-menopausal) were performed to evaluate whether risk of invasive breast cancer associated with treated hypertension, diuretic, and other AH medication use was modified by BMI or menopausal status (Table 3). A history of hypertension treated with any AH medication was not significantly associated with invasive breast cancer among any of the BMI categories women $(\mathrm{RR}=1.12,95 \% \mathrm{CI} 0.98-1.27$; $\mathrm{RR}=0.95,95 \%$ CI $0.81-1.11 ; \mathrm{RR}=1.09$, 95\% CI 0.90-1.32, for BMI $<25,25$ to $<30$, and $\geq 30 \mathrm{~kg} / \mathrm{m}^{2}$, respectively). Five or more years of diuretic use was significantly associated with risk of breast cancer only among women in the highest BMI category $(\mathrm{RR}=1.12,95 \% \mathrm{CI} 0.93-1.37 ; \mathrm{RR}=1.05$, 95\% CI 0.85-1.30; RR $=1.31,95 \%$ CI 1.03-1.66, for BMI $<25,25$ to $<30$, and $\geq 30 \mathrm{~kg} / \mathrm{m}^{2}$, respectively), and at least 5 years of use of "other" AH medications was associated with invasive breast cancer risk among women in the leanest and heaviest strata of BMI $(\mathrm{RR}=1.20,95 \% \mathrm{CI}$ 1.01-1.42; $\mathrm{RR}=1.00,95 \%$ CI $0.82-1.22 ; \mathrm{RR}=1.35$, $1.08-1.69$, for $\mathrm{BMI}<25,25$ to $<30$, and $\geq 30 \mathrm{~kg} / \mathrm{m}^{2}$, 
Table 1 Selected baseline characteristics and associations with a history of hypertension of 114,549 women in the California Teachers Study

\begin{tabular}{|c|c|c|c|c|c|c|c|c|c|c|}
\hline \multirow[t]{2}{*}{ Characteristic } & \multicolumn{3}{|l|}{ All women } & \multicolumn{3}{|c|}{ Women without hypertension } & \multicolumn{3}{|c|}{ Women with hypertension } & \multirow{2}{*}{$\begin{array}{l}\text { Age-adjusted } \\
p \text {-value }^{\mathrm{a}}\end{array}$} \\
\hline & $\begin{array}{l}\text { Mean } \\
\text { (SD) }\end{array}$ & $n$ & $\%$ & $\begin{array}{l}\text { Mean } \\
\text { (SD) }\end{array}$ & $n$ & $\%$ & $\begin{array}{l}\text { Mean } \\
(\mathrm{SD})\end{array}$ & $n$ & $\%$ & \\
\hline Age [years] & $52.8(13.9)$ & & & $50.9(13.4)$ & & & $62.2(12.1)$ & & & $<0.0001$ \\
\hline Race/ethnicity & & & & & & & & & & $<0.0001$ \\
\hline White & & 98,997 & 86.4 & & 82,480 & 86.6 & & 16,517 & 85.6 & \\
\hline $\begin{array}{l}\text { Non-White } \\
\quad \text { (includes unknown) }\end{array}$ & & 15,552 & 13.6 & & 12,767 & 13.4 & & 2,785 & 14.4 & \\
\hline $\begin{array}{l}\text { Family history of } \\
\text { breast cancer }\end{array}$ & & & & & & & & & & 0.01 \\
\hline None & & 97,393 & 85.0 & & 81,312 & 85.4 & & 16,081 & 83.3 & \\
\hline \multicolumn{11}{|c|}{ At least one affected first degree } \\
\hline Relative & & 13,484 & 11.8 & & 11,024 & 11.6 & & 2,460 & 12.7 & \\
\hline Unknown & & 3,672 & 3.2 & & 2,911 & 3.0 & & 761 & 3.9 & \\
\hline Body mass index $\left(\mathrm{kg} / \mathrm{m}^{2}\right)$ & & & & & & & & & & $<0.0001$ \\
\hline$<20$ & & 12,164 & 10.6 & & 11,170 & 11.7 & & 994 & 5.2 & \\
\hline 20 to $<25$ & & 55,121 & 48.1 & & 48,602 & 51.0 & & 6,519 & 33.8 & \\
\hline 25 to $<30$ & & 27,393 & 23.9 & & 21,660 & 22.7 & & 5,733 & 29.7 & \\
\hline 30 to $<35$ & & 10,046 & 8.8 & & 7,101 & 7.5 & & 2,945 & 15.3 & \\
\hline$\geq 35$ & & 5,589 & 4.9 & & 3,558 & 3.7 & & 2,031 & 10.5 & \\
\hline Unknown & & 4,236 & 3.7 & & 3,156 & 3.3 & & 1,080 & 5.6 & \\
\hline \multicolumn{11}{|l|}{ Hypertension } \\
\hline Never & & 95,247 & 83.1 & & 95,247 & 100.0 & & 0 & 0.0 & \\
\hline Ever & & 19,302 & 16.8 & & 0 & 0.0 & & 19,302 & 100.0 & \\
\hline \multicolumn{11}{|l|}{ Regular diuretic use } \\
\hline No & & 103,123 & 90.0 & & 92,568 & 97.2 & & 10,555 & 54.7 & \\
\hline Yes & & 11,426 & 10.0 & & 2,679 & 2.8 & & 8,747 & 45.3 & \\
\hline \multicolumn{11}{|l|}{ Regular reserpine use } \\
\hline No & & 114,049 & 99.6 & & 95,178 & 99.9 & & 18,871 & 97.8 & \\
\hline Yes & & 500 & 0.4 & & 69 & 0.1 & & 431 & 2.2 & \\
\hline \multicolumn{11}{|c|}{$\begin{array}{l}\text { Regular other antihypertensive } \\
\text { medication use }\end{array}$} \\
\hline No & & 99,685 & 87.0 & & 93,589 & 98.3 & & 6,096 & 31.6 & \\
\hline Yes & & 14,864 & 13.0 & & 1,658 & 1.7 & & 13,206 & 68.4 & \\
\hline Diabetes & & & & & & & & & & $<0.0001$ \\
\hline Never & & 111,367 & 97.2 & & 93,607 & 98.3 & & 17,760 & 92.0 & \\
\hline Ever & & 3,182 & 2.8 & & 1,640 & 1.7 & & 1,542 & 8.0 & \\
\hline Smoking history & & & & & & & & & & 0.10 \\
\hline Never & & 75,682 & 66.1 & & 63,936 & 67.1 & & 11,746 & 60.8 & \\
\hline Former & & 32,374 & 28.3 & & 25,981 & 27.3 & & 6,393 & 33.1 & \\
\hline Current & & 5,828 & 5.1 & & 4,778 & 5.0 & & 1,050 & 5.4 & \\
\hline Unknown & & 665 & 0.6 & & 552 & 0.6 & & 113 & 0.6 & \\
\hline $\begin{array}{l}\text { Alcohol use, average per } \\
\text { day in prior year (g/day) }\end{array}$ & & & & & & & & & & $<0.0001$ \\
\hline No use & & 36,463 & 31.8 & & 29,541 & 31.0 & & 6,922 & 35.9 & \\
\hline$<20$ & & 63,184 & 55.2 & & 53,663 & 56.3 & & 9,521 & 49.3 & \\
\hline $20+$ & & 8,986 & 7.8 & & 7,143 & 7.5 & & 1,843 & 9.6 & \\
\hline Unknown & & 5,916 & 5.2 & & 4,900 & 5.1 & & 1,016 & 5.3 & \\
\hline
\end{tabular}


Table 1 continued

\begin{tabular}{|c|c|c|c|c|c|c|c|c|c|c|}
\hline \multirow[t]{2}{*}{ Characteristic } & \multicolumn{3}{|c|}{ All women } & \multicolumn{3}{|c|}{ Women without hypertension } & \multicolumn{3}{|c|}{ Women with hypertension } & \multirow{2}{*}{$\begin{array}{l}\text { Age-adjusted } \\
p \text {-value }^{\mathrm{a}}\end{array}$} \\
\hline & $\begin{array}{l}\text { Mean } \\
\text { (SD) }\end{array}$ & $n$ & $\%$ & $\begin{array}{l}\text { Mean } \\
(\mathrm{SD})\end{array}$ & $n$ & $\%$ & $\begin{array}{l}\text { Mean } \\
(\mathrm{SD})\end{array}$ & $n$ & $\%$ & \\
\hline $\begin{array}{l}\text { Moderate/Strenuous } \\
\text { physical activity } \\
\text { (average lifetime) (h/week) }\end{array}$ & & & & & & & & & & $<0.0001$ \\
\hline$<2$ & & 36,148 & 31.6 & & 28,331 & 29.7 & & 7,817 & 40.5 & \\
\hline 2 to $<4$ & & 29,075 & 25.4 & & 24,413 & 25.6 & & 4,662 & 24.2 & \\
\hline 4 to $<6$ & & 19,168 & 16.7 & & 16,429 & 17.2 & & 2,739 & 14.2 & \\
\hline $6+$ & & 29,492 & 25.8 & & 25,577 & 26.8 & & 3,915 & 20.3 & \\
\hline Unknown & & 666 & 0.6 & & 497 & 0.5 & & 169 & 0.9 & \\
\hline $\begin{array}{l}\text { Age at first full-term } \\
\text { pregnancy (FFTP) }\end{array}$ & & & & & & & & & & $<0.0001$ \\
\hline FFTP age $<25$ & & 29,695 & 25.9 & & 23,380 & 24.6 & & 6,315 & 32.7 & \\
\hline FFTP age $25-29$ & & 33,465 & 29.2 & & 27,810 & 29.2 & & 5,655 & 29.3 & \\
\hline $\begin{array}{l}\text { No full-term pregnancies } \\
\text { prior to age } 30\end{array}$ & & 42,359 & 37.0 & & 36,304 & 38.1 & & 6,055 & 31.4 & \\
\hline Unknown & & 9,030 & 7.9 & & 7,753 & 8.1 & & 1,277 & 6.6 & \\
\hline Breastfeeding history & & & & & & & & & & $<0.0001$ \\
\hline $\begin{array}{l}\text { Never or less than } \\
12 \text { months }\end{array}$ & & 83,774 & 73.1 & & 68,504 & 71.9 & & 15,270 & 79.1 & \\
\hline $12+$ months & & 28,133 & 24.6 & & 24,675 & 25.9 & & 3,458 & 17.9 & \\
\hline Unknown & & 2,642 & 2.3 & & 2,068 & 2.2 & & 574 & 3.0 & \\
\hline Hysterectomy & & & & & & & & & & $<0.0001$ \\
\hline No & & 85,953 & 75.0 & & 74,482 & 78.2 & & 11,471 & 59.4 & \\
\hline Yes & & 26,179 & 22.8 & & 18,770 & 19.7 & & 7,409 & 38.4 & \\
\hline Unknown & & 2,417 & 2.1 & & 1,995 & 2.1 & & 422 & 2.2 & \\
\hline $\begin{array}{l}\text { Menopausal status and } \\
\text { hormone therapy use }\end{array}$ & & & & & & & & & & $<0.0001$ \\
\hline Premenopausal & & 46,927 & 41.0 & & 44,179 & 46.4 & & 2,748 & 14.2 & \\
\hline Peri-menopausal & & 2,361 & 2.1 & & 2,012 & 2.1 & & 349 & 1.8 & \\
\hline $\begin{array}{c}\text { Post-menopausal, } \\
\text { never HT use }\end{array}$ & & 12,015 & 10.5 & & 8,668 & 9.1 & & 3,347 & 17.3 & \\
\hline $\begin{array}{l}\text { Post-menopausal, } \\
\text { any HT use }\end{array}$ & & 39,379 & 34.4 & & 29,034 & 30.5 & & 10,345 & 53.6 & \\
\hline Unknown & & 13,867 & 12.1 & & 11,354 & 11.9 & & 2,513 & 13.0 & \\
\hline $\begin{array}{l}\text { Adherence to screening } \\
\text { guidelines }\end{array}$ & & & & & & & & & & $<0.0001$ \\
\hline No & & 41,109 & 35.9 & & 33,347 & 35.0 & & 7,762 & 40.2 & \\
\hline Yes & & 48,911 & 42.7 & & 38,942 & 40.9 & & 9,969 & 51.6 & \\
\hline Unknown & & 24,529 & 21.4 & & 22,958 & 24.1 & & 1,571 & 8.1 & \\
\hline $\begin{array}{l}\text { Percent calories from fat, } \\
\text { excluding alcohol } \\
\text { (quartiles) }\end{array}$ & & & & & & & & & & $<0.0001$ \\
\hline First & & 26,064 & 22.8 & & 21,718 & 22.8 & & 4,346 & 22.5 & \\
\hline Second & & 26,077 & 22.8 & & 22,088 & 23.2 & & 3,989 & 20.7 & \\
\hline Third & & 26,062 & 22.8 & & 21,885 & 23.0 & & 4,177 & 21.6 & \\
\hline Fourth & & 26,106 & 22.8 & & 21,290 & 22.4 & & 4,816 & 25.0 & \\
\hline Unknown & & 10,240 & 8.9 & & 8,266 & 8.7 & & 1,974 & 10.2 & \\
\hline
\end{tabular}

${ }^{a} P$-value for association with hypertension calculated using logistic regression adjusting for age at baseline

${ }^{\mathrm{b}}$ For women age $\geq 40$, mammogram within 1 year of baseline; for women age $<40$, breast exam by health care provider within 2 years or mammogram within 1 year of baseline 
Table 2 Relative risk estimates for association between hypertension, antihypertensive (AH) medication use, and invasive breast cancer overall and for estrogen receptor (ER) subtypes in baseline respondents in the California Teachers Study

\begin{tabular}{|c|c|c|c|c|c|c|c|c|}
\hline \multirow[t]{2}{*}{ Variable } & \multirow{2}{*}{$\begin{array}{l}\text { Observed } \\
\text { person-years }\end{array}$} & \multicolumn{3}{|c|}{ Invasive breast cancer } & \multicolumn{2}{|c|}{$\mathrm{ER}+$ breast cancer } & \multicolumn{2}{|c|}{ ER - breast cancer } \\
\hline & & $\begin{array}{l}\text { No. of } \\
\text { cases }^{b}\end{array}$ & $\begin{array}{l}\text { Age-adjusted } \\
\text { RR (95\% CI) }\end{array}$ & $\begin{array}{l}\text { Multivariable- } \\
\text { adjusted RR } \\
(95 \% \mathrm{CI})^{\mathrm{a}}\end{array}$ & $\begin{array}{l}\text { No. of } \\
\text { cases }\end{array}$ & $\begin{array}{l}\text { Multivariable- } \\
\text { adjusted RR } \\
(95 \% \mathrm{CI})\end{array}$ & $\begin{array}{l}\text { No. of } \\
\text { cases }\end{array}$ & $\begin{array}{l}\text { Multivariable- } \\
\text { adjusted RR } \\
(95 \% \text { CI })\end{array}$ \\
\hline \multicolumn{9}{|c|}{ Treated hypertension } \\
\hline Never & 991,013 & 3,341 & 1.0 (ref) & 1.0 (ref) & 2,443 & 1.0 (ref) & 453 & 1.0 (ref) \\
\hline Ever & 155,427 & 810 & $1.06(0.98-1.14)$ & $1.06(0.98-1.16)$ & 585 & $1.09(0.99-1.20)$ & 91 & $0.98(0.78-1.25)$ \\
\hline \multicolumn{9}{|c|}{ Minimum duration of any $\mathrm{AH}$ medication use } \\
\hline No regular use & 954,624 & 31,79 & 1.0 (ref) & 1.0 (ref) & 2,312 & 1.0 (ref) & 432 & 1.0 (ref) \\
\hline$\leq 1$ year & 44,076 & 171 & $0.90(0.77-1.05)$ & $0.90(0.77-1.06)$ & 120 & $0.87(0.72-1.05)$ & 23 & $0.92(0.60-1.41)$ \\
\hline $2-4$ years & 55,797 & 256 & $0.99(0.87-1.13)$ & $1.00(0.88-1.14)$ & 184 & $0.99(0.85-1.15)$ & 25 & $0.78(0.51-1.16)$ \\
\hline $5-9$ years & 37,072 & 212 & $1.18(1.02-1.36)$ & $1.18(1.02-1.36)$ & 159 & $1.21(1.03-1.43)$ & 27 & $1.19(0.80-1.78)$ \\
\hline $10+$ years & 50,588 & 305 & $1.17(1.04-1.32)$ & $\underline{1.18(1.04-1.34)}$ & 237 & $\underline{1.26(1.10-1.45)}$ & 31 & $1.00(0.69-1.47)$ \\
\hline \multicolumn{9}{|c|}{ Regular diuretic use } \\
\hline No & $1,038,514$ & 3,598 & 1.0 (ref) & 1.0 (ref) & 2,628 & 1.0 (ref) & 477 & 1.0 (ref) \\
\hline Yes & 107,926 & 553 & $1.05(0.96-1.16)$ & $1.06(0.96-1.16)$ & 400 & $1.04(0.93-1.16)$ & 67 & $1.06(0.81-1.38)$ \\
\hline \multicolumn{9}{|c|}{ Duration of diuretic use } \\
\hline No regular use & $1,038,514$ & 3,598 & 1.0 (ref) & 1.0 (ref) & 2,628 & 1.0 (ref) & 477 & 1.0 (ref) \\
\hline$\leq 1$ year & 21,734 & 86 & $0.90(0.73-1.12)$ & $0.91(0.74-1.13)$ & 59 & $0.85(0.66-1.10)$ & 13 & $1.08(0.62-1.87)$ \\
\hline $2-4$ years & 26,642 & 120 & $0.97(0.81-1.16)$ & $0.97(0.81-1.17)$ & 84 & $0.92(0.74-1.15)$ & 11 & $0.72(0.40-1.32)$ \\
\hline $5-9$ years & 19,767 & 109 & $1.11(0.92-1.35)$ & $1.11(0.92-1.35)$ & 76 & $1.05(0.84-1.33)$ & 16 & $1.34(0.81-2.22)$ \\
\hline $10+$ years & 37,804 & 227 & $1.16(1.01-1.32)$ & $1.16(1.01-1.33)$ & 175 & $1.21(1.03-1.42)$ & 25 & $1.11(0.73-1.67)$ \\
\hline \multicolumn{9}{|c|}{ Regular other AH medication use } \\
\hline No & $1,005,301$ & 3,424 & 1.0 (ref) & 1.0 (ref) & 2,501 & 1.0 (ref) & 456 & 1.0 (ref) \\
\hline Yes & 141,139 & 727 & $1.07(0.99-1.17)$ & $1.08(0.99-1.18)$ & 527 & $1.07(0.97-1.18)$ & 88 & $1.06(0.84-1.35)$ \\
\hline \multicolumn{9}{|c|}{ Duration of other $\mathrm{AH}$ medication use } \\
\hline No regular use & $1,005,301$ & 3,424 & 1.0 (ref) & 1.0 (ref) & 2,501 & 1.0 (ref) & 456 & 1.0 (ref) \\
\hline$\leq 1$ year & 25,032 & 96 & $0.89(0.73-1.10)$ & $0.89(0.73-1.10)$ & 66 & $0.84(0.66-1.08)$ & 13 & $0.94(0.54-1.63)$ \\
\hline $2-4$ years & 38,929 & 183 & $1.02(0.87-1.18)$ & $1.02(0.88-1.18)$ & 129 & $0.99(0.83-1.18)$ & 19 & $0.84(0.53-1.34)$ \\
\hline $5-9$ years & 30,015 & 168 & $1.16(0.99-1.35)$ & $1.16(0.99-1.36)$ & 127 & $1.19(0.99-1.43)$ & 19 & $1.06(0.67-1.69)$ \\
\hline $10+$ years & 42,998 & 253 & $1.14(1.00-1.30)$ & $1.15(1.01-1.31)$ & 188 & $1.17(1.00-1.36)$ & 31 & $1.20(0.82-1.76)$ \\
\hline
\end{tabular}

RRs in bold have $p$-values $<0.05$, and RRs underlined have $p$-values $<0.01$

a Age-stratified analyses adjusted for categories of race, family history of breast cancer, age at first full-term pregnancy and number of full-term pregnancies combined variable, hormone therapy and menopausal status combined variable, lifetime physical activity, diabetes, body mass index, smoking history, alcohol use, hysterectomy, breastfeeding, and quartiles of percent calories from fat

${ }^{b}$ Person-years and number of cases may not add up to total due to rounding or unknown values

respectively) and among pre-/peri-menopausal women $(\mathrm{RR}=1.58,95 \%$ CI $1.11-2.25)$, but not among postmenopausal women ( $\mathrm{RR}=1.10,95 \%$ CI $0.98-1.24)$.

We were able to explore the effects of specific $\mathrm{AH}$ medications based on data collected during the 2000-2001 survey. Associations between reported AH medication use in the two years preceding the 2000-2001 survey and breast cancer risk are presented in Table 4. Use of $\mathrm{AH}$ medications including thiazide or furosemide diuretics, calcium channel blockers, ACE inhibitors, or other antihypertensive medications was not significantly associated with invasive breast cancer risk.

\section{Discussion}

In this large prospective cohort study, long-term use of $\mathrm{AH}$ medication was associated with increased risk of invasive breast cancer, and this risk appeared to be confined to ERpositive tumors, and among pre-/peri-menopausal women and those who were in the lowest or highest categories of BMI.

While there is fairly consistent evidence linking hypertension and antihypertensive medications to renal cancer [7] and hypertension to endometrial cancer [5, 7, 8, 17], prior published studies of a hypertension-breast cancer 
Table 3 Relative risk estimates for association between hypertension, antihypertensive (AH) medication use, and invasive breast cancer by strata of body mass index (BMI) and menopausal status in baseline respondents in the California Teachers Study

\begin{tabular}{|c|c|c|c|c|c|c|c|c|c|}
\hline \multirow[t]{2}{*}{ Strata } & \multirow[t]{2}{*}{$\begin{array}{l}\text { Breast } \\
\text { cancer } \\
\text { cases, No. }\end{array}$} & \multicolumn{2}{|c|}{$\begin{array}{l}\text { History of treated } \\
\text { hypertension, } \mathrm{RR}^{\mathrm{a}} \\
(95 \% \mathrm{CI})\end{array}$} & \multicolumn{3}{|c|}{ Diuretic use, $\mathrm{RR}^{\mathrm{a}}(95 \% \mathrm{CI})$} & \multicolumn{3}{|c|}{$\begin{array}{l}\text { Other antihypertensive } \\
\text { medication use, } \mathrm{RR}^{\mathrm{a}}(95 \% \mathrm{CI})\end{array}$} \\
\hline & & No & Yes & $\begin{array}{l}\text { No } \\
\text { regular use }\end{array}$ & $<5$ years of use & $5+$ years of use & $\begin{array}{l}\text { No } \\
\text { regular use }\end{array}$ & $<5$ years of use & $5+$ years of use \\
\hline \multicolumn{10}{|l|}{ BMI } \\
\hline$<25$ & 2291 & 1.0 (ref) & $1.12(0.98-1.27)$ & 1.0 (ref) & $0.77(0.59-1.00)$ & $1.12(0.93-1.37)$ & 1.0 (ref) & $1.01(0.83-1.23)$ & $1.20(1.01-1.42)$ \\
\hline $25-29$ & 1127 & 1.0 (ref) & $0.95(0.81-1.11)$ & 1.0 (ref) & $1.00(0.78-1.28)$ & $1.05(0.85-1.30)$ & 1.0 (ref) & $0.92(0.73-1.16)$ & $1.00(0.82-1.22)$ \\
\hline $30+$ & 569 & 1.0 (ref) & $1.09(0.90-1.32)$ & 1.0 (ref) & $1.08(0.80-1.45)$ & $1.31(1.03-1.66)$ & 1.0 (ref) & $0.89(0.66-1.19)$ & $1.35(1.08-1.69)$ \\
\hline \multicolumn{10}{|c|}{ Menopausal status } \\
\hline $\begin{array}{l}\text { Pre/peri- } \\
\text { menopausal }\end{array}$ & 1016 & 1.0 (ref) & $1.08(0.83-1.41)$ & 1.0 (ref) & $0.82(0.53-1.25)$ & $1.13(0.70-1.81)$ & 1.0 (ref) & $0.90(0.63-1.28)$ & $1.58(1.11-2.25)$ \\
\hline $\begin{array}{l}\text { Post- } \\
\text { menopausal }\end{array}$ & 2760 & 1.0 (ref) & $1.05(0.96-1.15)$ & 1.0 (ref) & $0.94(0.80-1.11)$ & $1.13(1.00-1.28)$ & 1.0 (ref) & $0.96(0.83-1.10)$ & $1.10(0.98-1.24)$ \\
\hline
\end{tabular}

${ }^{a}$ Age-stratified analyses adjusted for categories of race, family history of breast cancer, age at first full-term pregnancy and number of full-term pregnancies combined variable, lifetime physical activity, diabetes, smoking history, alcohol use, breastfeeding, and quartiles of percent calories from fat: analyses stratified by BMI were further adjusted for hysterectomy, menopausal status, and hormone therapy use combined variable, and analyses stratified by menopausal status were further adjusted for BMI

RRs in bold have $p$-values $<0.05$

association have been inconsistent $[7,8,18-20]$. Some prior studies included only women aged 50 and older [18, 19], while others included younger women as well $[8,20]$. In the present study, we found the AH medication-breast cancer association was most pronounced in younger women. However, a study by Soler et al. [8] stratified by age found the risk to be confined to post-menopausal women, and two additional studies reported significant hypertension and breast cancer associations among women above age 50 at enrollment $[18,19]$. Furthermore, with a fairly long list of potential or established shared risk factors, it is possible that differential or inadequate adjustment for such factors may also account for some of the inconsistencies. For example, not all prior studies accounted for alcohol use [18], lifetime physical activity [8, 18-20], breastfeeding $[8,18,19]$, hormone therapy use $[8,18,19]$, hysterectomy [8, 18-20], or diabetes [8, 18]. Also, because the magnitude of any true association between hypertension, AH medications, and breast cancer is likely weak, there may be issues of inadequate power for smaller studies. Studies which have stratified by BMI have tended to report stronger associations of hypertension with breast cancer among women with higher BMI [8, 19]. Stratification by BMI in the present study indicated that at least 5 years of use of other $\mathrm{AH}$ medication was associated with invasive breast cancer among women in both the lowest $\left(<25 \mathrm{~kg} / \mathrm{m}^{2}\right)$ and highest $\left(\geq 30 \mathrm{~kg} / \mathrm{m}^{2}\right)$ categories of BMI, but not for women in the middle category of BMI (25 to $<30 \mathrm{~kg} / \mathrm{m}^{2}$ ), and at least 5 years of diuretic use was associated with invasive breast cancer only among women in the highest $\left(\geq 30 \mathrm{~kg} / \mathrm{m}^{2}\right)$ category of BMI. Furthermore, when analyses were stratified by both menopausal status and BMI (results not shown), associations between treated hypertension, AH medication use, and invasive breast cancer risk were confined to pre-/peri-menopausal women in the highest category of BMI.

An important challenge for research investigating the interrelationships between hypertension, AH medications, and breast cancer risk is that of disentangling the independent effects of the condition versus pharmacologic treatments. In a prospective study of hypertensive women which included measures of diastolic blood pressure (DBP) at baseline, increasing DBP was associated with increased risk of breast cancer among women who were not taking AH drugs but was inversely associated with risk among women taking AH drugs [21]. In the longitudinal Women's Health Initiative Study, increasing baseline DBP also was reported to be associated with a borderline increased risk of total breast cancer (invasive and in situ), but not invasive breast cancer alone [22]. A limitation of the present study is lack of information about whether hypertension was controlled by either AH medications or lifestyle modifications as well as a lack of information about specific types of medications beyond broad classes in the baseline questionnaire. Furthermore, with the exception of the antihypertensive medications queried on the 2000-2001 survey, both hypertension and $\mathrm{AH}$ medication use were recorded on the baseline survey, and any changes that may have occurred during years of follow-up could have resulted in some misclassification of exposure status. 
Table 4 Relative risk estimates for association between classes of antihypertensive medication use and invasive breast cancer among follow-up respondents in California Teachers Study

\begin{tabular}{|c|c|c|c|c|}
\hline Variable & $n$ & $\begin{array}{l}\text { Observed } \\
\text { person-years }\end{array}$ & $\begin{array}{l}\text { Invasive breast cancer } \\
\text { cases, no. }(1,714 \text { total })\end{array}$ & $\begin{array}{l}\text { Multivariable adjusted } \\
\text { RR }(95 \% \mathrm{CI})^{\mathrm{a}}\end{array}$ \\
\hline \multicolumn{5}{|c|}{ Any antihypertensive medication } \\
\hline No regular use & 56,710 & 361,865 & 1,206 & $1.0(\mathrm{ref})$ \\
\hline Regular use within 2 years & 17,032 & 103,558 & 508 & $1.09(0.98-1.22)$ \\
\hline \multicolumn{5}{|l|}{ Thiazide diuretic } \\
\hline No regular use & 68,409 & 432,781 & 1,540 & $1.0(\mathrm{ref})$ \\
\hline Regular use within 2 years & 5,324 & 32,583 & 172 & $1.16(0.99-1.37)$ \\
\hline Unknown & 9 & 59 & 2 & \\
\hline \multicolumn{5}{|l|}{ Furosemide diuretic (Lasix) } \\
\hline No regular use & 72,175 & 457,054 & 1,664 & 1.0 (ref) \\
\hline Regular use within 2 years & 1,562 & 8,350 & 50 & $1.22(0.91-1.65)$ \\
\hline (Unknown) & 5 & 19 & 0 & \\
\hline \multicolumn{5}{|l|}{ Calcium channel blocker } \\
\hline No regular use & 70,862 & 448,175 & 1,630 & 1.0 (ref) \\
\hline Regular use within 2 years & 2,873 & 17,208 & 84 & $1.05(0.84-1.31)$ \\
\hline Unknown & 7 & 40 & 0 & \\
\hline \multicolumn{5}{|l|}{ ACE inhibitor } \\
\hline No regular use & 69,698 & 440,762 & 1,592 & 1.0 (ref) \\
\hline Regular use within 2 years & 4,034 & 24,593 & 122 & $1.05(0.86-1.27)$ \\
\hline Unknown & 10 & 68 & 0 & \\
\hline \multicolumn{5}{|c|}{ Other antihypertensive medication } \\
\hline No regular use & 63,242 & 401,638 & 1,410 & 1.0 (ref) \\
\hline Regular use within 2 years & 10,441 & 63,428 & 303 & $1.04(0.91-1.18)$ \\
\hline Unknown & 59 & 357 & 1 & \\
\hline
\end{tabular}

${ }^{a}$ Age-stratified analyses adjusted for categories of race, family history of breast cancer, age at first full-term pregnancy and number of full-term pregnancies combined variable, hormone therapy and menopausal status combined variable, lifetime physical activity, diabetes, body mass index, smoking history, alcohol use, hysterectomy, breastfeeding, and quartiles of percent calories from fat

b Totals may not add up due to rounding

AH medications have been associated with risk of various malignancies. A pooled analysis of AH medication use and risk of malignancies revealed a significant association between rauwolfia derivatives, including reserpine, and breast cancer yielding a significant $25 \%$ increase in breast cancer risk [23]. This pooled analysis also supported an association between diuretics and increased risk of renal cell carcinoma while other AH drugs including ACE inhibitors and calcium antagonists were not found to be associated with cancer risk. In a case-control study among women aged 65-79, $\mathrm{Li}$ and colleagues [24] reported that thiazide diuretics (OR $=1.4,95 \% \mathrm{CI} 1.1-1.8)$, potassiumsparing diuretics $(\mathrm{OR}=1.6,95 \% \mathrm{CI} 1.2-2.1)$, and immediate release calcium channel blockers $(\mathrm{OR}=1.5,95 \% \mathrm{CI}$ 1.0-2.1) were associated with modest increased risk of invasive breast cancer, however, no trend of increasing risk with increasing duration of use was observed. It has been suggested that insulin resistance among thiazide diuretic users and increased insulin levels associated with use of potassium-sparing diuretics are potential mechanisms
[24, 25] which could explain an association between diuretic use and breast cancer. One additional [19] study among women aged 50-75, but not other previous studies [25-27], investigating the risk of breast cancer associated with diuretic use, have reported a significant association. It is not clear in some of the prior studies whether in situ breast cancers were included as outcomes [26, 27]. In the present study, an association between thiazide diuretic use and invasive breast cancer risk was not observed.

The observation in the present study of increased breast cancer risk with long-term use of $\mathrm{AH}$ medication raises a question as to whether it is the condition of hypertension itself or exposure to medications which may influence breast cancer risk. It is likely that long-term use of $\mathrm{AH}$ medication is a surrogate marker for longer duration of a hypertensive condition. However, certain AH medications may have been taken for other conditions, including cardiac arrhythmia (beta blockers, calcium channel blockers), coronary artery disease with or without history of myocardial infarction (beta blockers), congestive heart failure 
(ACE inhibitors, furosemide diuretics), diabetic nephropathy (ACE inhibitors), or off-label usage such as diuretics for weight loss. One hypothesis for why duration of exposure to the condition of hypertension may be an important factor in a hypertension-cancer association has been reviewed by Hamet [13] who postulates that with short-term exposure to hypertension, during the period in which hypertension is developing, apoptosis is increased which may be associated with reduced risk of cancer [28]. Long-term exposure, on the other hand, could be associated with increased cancer risk as it is associated with cellular senescence, telomere shortening [29], and inhibition of apoptosis. Other potential biologic mechanisms contributing to an association between hypertension and cancer include pathways shared in steroid hormone synthesis and metabolism [12], angiogenesis [30], growth factor signaling [31], and inflammation [22, 32].

Strengths of the current study include the prospective design where hypertension and $\mathrm{AH}$ medication use were assessed prior to follow-up for breast cancer incidence thus largely eliminating the potential for recall bias. However, because the primary exposures of interest, history of hypertension, and $\mathrm{AH}$ medication use were primarily assessed by self-report, the potential for misclassification exists. A recent NHANES report revealed that $87 \%$ of hypertensive women in the United States. are aware of their condition; further, less than $5 \%$ of women were hypertensive, but had never been diagnosed by a health care provider [33]. The sensitivity of self-reported antihypertensive medication use among older women has been reported to be 79-92\% [34]. Any such misclassification of AH medication is, however, expected to be non-differential with respect to breast cancer status, and thus our findings should if anything be an underestimate of the true effects.

The ascertainment of incident breast cancer diagnoses through linkage with the comprehensive population-based $\mathrm{CCR}$, rather than relying on self-reports, is another strength of our study as case ascertainment is $99 \%$ complete in the state of California [35].

The CTS cohort is also well characterized with respect to covariates of interest which allowed for adjustment for several potential confounding factors. While breast cancer and hypertension appear to share many common risk factors, the age-adjusted and multivariate-adjusted risk estimates were quite similar in this study, suggesting that the covariates did not have a major influence on the association between $\mathrm{AH}$ medications and breast cancer. However, these effects were rather moderate, and so we cannot exclude the possibility that residual confounding may account for the observed associations.

In summary, it is difficult to separate the independent effects of the condition of hypertension itself (and varying levels of severity and control) and treatment in the form of
AH medications on the risk of breast cancer. However, given the high prevalence of both hypertension and $\mathrm{AH}$ medication use, even small effects on breast cancer risk could potentially have population-wide implications. Future research into the biologic mechanisms may help disentangle the etiological roles of hypertension itself versus the use of specific antihypertensive medications in breast cancer risk.

Acknowledgments The authors would like to thank the other CTS Steering Committee members who are responsible for the formation and maintenance of the cohort within which this study was conducted but who did not directly contribute to the current paper: Ellen Chang, Christina Clarke, Rosemary Cress, Dennis Deapen, Katherine Henderson, David Nelson, Rich Pinder, Daniel Stram, and Dee West; and the women participating as members of the CTS cohort.

Financial support This work was supported by the National Institutes of Health [CA77398]. The collection of cancer incidence data used in this study was supported by the California Department of Public Health as part of the statewide cancer reporting program mandated by California Health and Safety Code Section 103885; the National Cancer Institute's Surveillance, Epidemiology and End Results Program under contract N01-PC-35136 awarded to the Northern California Cancer Center, contract N01-PC-35139 awarded to the University of Southern California, and contract N02-PC-15105 awarded to the Public Health Institute; and the Centers for Disease Control and Prevention's National Program of Cancer Registries, under agreement \#U55/CCR921930-02 awarded to the Public Health Institute. The ideas and opinions expressed herein are those of the authors and endorsement by the State of California, Department of Health Services, the National Cancer Institute, and the Centers for Disease Control and Prevention or their contractors and subcontractors is not intended nor should be inferred.

Open Access This article is distributed under the terms of the Creative Commons Attribution Noncommercial License which permits any noncommercial use, distribution, and reproduction in any medium, provided the original author(s) and source are credited.

\section{References}

1. Horner MJ RL, Krapcho M, Neyman N, Aminou R, Howlader N, Altekruse SF, Feuer EJ, Huang L, Mariotto A, Miller BA, Lewis DR, Eisner MP, Stinchcomb DG, Edwards BK (eds) (2009) SEER Cancer Statistics Review, 1975-2006. In: based on November 2008 SEER data submission. National Cancer Institute, Bethesda

2. Surveillance E, End Results (SEER) Program (www.seer. cancer.gov) SEER*Stat Databasev (2008) Mortality-All COD, Public-Use with State, Total U.S. (1969-2005), National Cancer Institute, DCCPS, Surveillance Research Program, Cancer Statistics Branch, released February 2008

3. Burt VL, Whelton P, Roccella EJ, Brown C, Cutler JA, Higgins M, Horan MJ, Labarthe D (1995) Prevalence of hypertension in the US adult population. Results from the Third National Health and Nutrition Examination Survey, 1988-1991. Hypertension 25(3):305-313

4. Corrao G, Scotti L, Bagnardi V, Sega R (2007) Hypertension, antihypertensive therapy and renal-cell cancer: a meta-analysis. Curr Drug Saf 2(2):125-133

5. Furberg AS, Thune I (2003) Metabolic abnormalities (hypertension, hyperglycemia and overweight), lifestyle (high energy 
intake and physical inactivity) and endometrial cancer risk in a Norwegian cohort. Int J Cancer 104(6):669-676

6. Boynton-Jarrett R, Rich-Edwards J, Malspeis S, Missmer SA, Wright R (2005) A prospective study of hypertension and risk of uterine leiomyomata. Am J Epidemiol 161(7):628-638

7. Lindgren AM, Nissinen AM, Tuomilehto JO, Pukkala E (2005) Cancer pattern among hypertensive patients in North Karelia, Finland. J Hum Hypertens 19(5):373-379

8. Soler M, Chatenoud L, Negri E, Parazzini F, Franceschi S, la Vecchia C (1999) Hypertension and hormone-related neoplasms in women. Hypertension 34(2):320-325

9. Goon PK, Messerli FH, Lip GY (2006) Hypertension and breast cancer: an association revisited? J Hum Hypertens 20(10):722724

10. Schwarz EB, Ray RM, Stuebe AM, Allison MA, Ness RB, Freiberg MS, Cauley JA (2009) Duration of lactation and risk factors for maternal cardiovascular disease. Obstet Gynecol 113(5):974-982

11. Vona-Davis L, Howard-McNatt M, Rose DP (2007) Adiposity, type 2 diabetes and the metabolic syndrome in breast cancer. Obes Rev 8(5):395-408

12. Xing D, Nozell S, Chen YF, Hage F, Oparil S (2009) Estrogen and mechanisms of vascular protection. Arterioscler Thromb Vasc Biol 29(3):289-295

13. Hamet P (1997) Cancer and hypertension: a potential for crosstalk? J Hypertens 15(12 Pt 2):1573-1577

14. Giordano SH (2003) Breast carcinoma and antihypertensive therapy. Cancer 98(7):1334-1336

15. Bernstein L, Allen M, Anton-Culver H, Deapen D, Horn-Ross PL, Peel D, Pinder R, Reynolds P, Sullivan-Halley J, West D et al (2002) High breast cancer incidence rates among California teachers: results from the California Teachers Study (United States). Cancer Causes Control 13(7):625-635

16. Kelsey JL, Whittemore AS, Evans AS (1986) Methods in observational epidemiology. Oxford University Press, Oxford

17. Weiderpass E, Persson I, Adami HO, Magnusson C, Lindgren A, Baron JA (2000) Body size in different periods of life, diabetes mellitus, hypertension, and risk of postmenopausal endometrial cancer (Sweden). Cancer Causes Control 11(2):185-192

18. Peeters PH, van Noord PA, Hoes AW, Fracheboud J, Gimbrere $\mathrm{CH}, \mathrm{DE}$ Grobbee (2000) Hypertension and breast cancer risk in a 19-year follow-up study (the DOM cohort). Diagnostic investigation into mammarian cancer. J Hypertens 18(3):249-254

19. Largent JA, McEligot AJ, Ziogas A, Reid C, Hess J, Leighton N, Peel D, Anton-Culver H (2006) Hypertension, diuretics and breast cancer risk. J Hum Hypertens 20(10):727-732

20. Beji NK, Reis N (2007) Risk factors for breast cancer in Turkish women: a hospital-based case-control study. Eur J Cancer Care (Engl) 16(2):178-184

21. Lindgren A, Pukkala E, Tuomilehto J, Nissinen A (2007) Incidence of breast cancer among postmenopausal, hypertensive women. Int J Cancer 121(3):641-644
22. Kabat GC, Kim M, Chlebowski RT, Khandekar J, Ko MG, McTiernan A, Neuhouser ML, Parker DR, Shikany JM, Stefanick ML et al (2009) A longitudinal study of the metabolic syndrome and risk of postmenopausal breast cancer. Cancer Epidemiol Biomarkers Prev 18(7):2046-2053

23. Grossman E, Messerli FH, Goldbourt U (2001) Antihypertensive therapy and the risk of malignancies. Eur Heart J 22(15): $1343-1352$

24. Li CI, Malone KE, Weiss NS, Boudreau DM, Cushing-Haugen KL, Daling JR (2003) Relation between use of antihypertensive medications and risk of breast carcinoma among women ages 65-79 years. Cancer 98(7):1504-1513

25. Coogan PF, Strom BL, Rosenberg L (2009) Diuretic use and the risk of breast cancer. J Hum Hypertens 23(3):216-218

26. Fryzek JP, Poulsen AH, Lipworth L, Pedersen L, Norgaard M, McLaughlin JK, Friis S (2006) A cohort study of antihypertensive medication use and breast cancer among Danish women. Breast Cancer Res Treat 97(3):231-236

27. Gonzalez-Perez A, Ronquist G, Garcia Rodriguez LA (2004) Breast cancer incidence and use of antihypertensive medication in women. Pharmacoepidemiol Drug Saf 13(8):581-585

28. Hamet P, Richard L, Dam TV, Teiger E, Orlov SN, Gaboury L, Gossard F, Tremblay J (1995) Apoptosis in target organs of hypertension. Hypertension 26(4):642-648

29. Aviv A, Aviv H (1997) Reflections on telomeres, growth, aging, and essential hypertension. Hypertension 29(5):1067-1072

30. Sane DC, Anton L, Brosnihan KB (2004) Angiogenic growth factors and hypertension. Angiogenesis 7(3):193-201

31. Hunt KJ, Lukanova A, Rinaldi S, Lundin E, Norat T, Palmqvist R, Stattin P, Riboli E, Hallmans G, Kaaks R (2006) A potential inverse association between insulin-like growth factor I and hypertension in a cross-sectional study. Ann Epidemiol 16(7): 563-571

32. Lithgow D, Covington C (2005) Chronic inflammation and breast pathology: a theoretical model. Biol Res Nurs 7(2):118-129

33. Ostchega Y, Yoon SS, Hughes J, Louis T (2008) Hypertension awareness, treatment, and control-continued disparities in adults: United States, 2005-2006. In: NCHS data brief. National Center for Health Statistics, Hyattsville

34. Boudreau DM, Daling JR, Malone KE, Gardner JS, Blough DK, Heckbert SR (2004) A validation study of patient interview data and pharmacy records for antihypertensive, statin, and antidepressant medication use among older women. Am J Epidemiol 159(3):308-317

35. Kwong S PC, Morris C, Cohen R, Allen M, Wright W (2001) Cancer in California: 1988-1999. In: Sacramento: California Department of Health Services, Cancer Surveillance Section 